\title{
World Journal of Emergency Surgery reviewer acknowledgement 2015
}

Fausto Catena $^{1^{*}}$ and Frederick Moore ${ }^{2}$

\section{Contributing reviewers}

The editors of World Journal of Emergency Surgery would like to thank all our reviewers who have contributed to the journal in volume 10 (2015).

Fikri Abu-Zidan

United Arab Emirates

Ferdinando Agresta

Italy

Jamir Arlikar

India

Erman Aytac

Turkey

Miklosh Bala

Israel

Cino Bendinelli

Australia

Jasneet Bhullar

United States of America

Walter Biffl

United States of America

Luigi Bonavina

Italy

Clay Cothren Burlew

United States of America

Fabio Campanile

Italy

Osvaldo Chiara

Italy
Mircea Chirica
France

Ian Civil

New Zealand

Federico Coccolini

Italy

Raul Coimbra

United States of America

Lidia Dalfino

Italy

James Davis

United States of America

Marc De Moya

United States of America

Nicola de'Angelis

France

Marc deMoya

United States of America

Salomone Di Saverio

Italy

Jose Diaz

United States of America

Paul Eustace

Ireland

\section{Eriberto Farinella \\ Italy}

Paula Ferrada

United States of America

Andreas Fette

Germany

Gustavo Fraga

Ralf Herbert Gahr

Germany

Sanjay Gupta

India

Daithi Heffernan

United States of America

Rao Ivatury

United States of America

Jeffry Kashuk

Israel

Radwan Kassir

France

Kenji Kawamukai

Italy

Michael Kelly

Australia

\footnotetext{
* Correspondence: faustocatena@gmail.com

'Department of Emergency and General Surgery, Parma University Hospital,

Parma, Italy

${ }^{2}$ Department of Surgery, University of Florida, Gainesville, Florida, USA
} 
Peter Kim

United States of America

Fernando Kim

United States of America

Boris Kirshtein

Israel

Yoram Kluger

Israel

Rosemary Kozar

United States of America

Rifat Latifi

United States of America

I. Michael Leitman

United States of America

Ari Leppaniemi

Finland

Lawrence Lottenberg

United States of America

Ronald Maier

United States of America

Mark Malangoni

United States of America

Ingo Marzi

Germany

Oner Mentes

Turkey
David Muckart

South Africa

Pradeep Navsaria

South Africa

Vinesh Padayachy

South Africa

Abhijeet Patil

United Kingdom

Edoardo Picetti

Italy

Stefano Rausei

Italy

Joao Rezende-Neto

Canada

Sandro Rizoli

Canada

Giorgio Rossi

Italy

Boris Sakakushev

Bulgaria

Massimo Sartelli

Italy

Thomas Scalea

United States of America

Helmut Segovia Lohse

Paraguay
Pablo Serrano

Canada

Mohd Sheikh

United States of America

Kjetil Soreide

Norway

Philip Stahel

United Kingdom

Angela Sultana

Malta

Peep Talving

Estonia

Gia Tomadze

Georgia

Gregorio Tugnoli

Italy

George Velmahos

United States of America

Imtiaz Wani

India

Dieter Weber

Australia 